Pesq. Vet. Bras. 35(3):241-248, março 2015 DOI: $10.1590 /$ S0100-736X2015000300006

\title{
Avaliação da resposta imunológica da mucosa intestinal de frangos de corte desafiados com diferentes sorovares de Salmonella ${ }^{1}$
}

\author{
Eduardo C. Muniz ${ }^{2 *}$, Larissa Pickler², Mariana C. Lourenço ${ }^{2}$, Antônio L. Kraieski² \\ Dany Mesa $^{2}$, Patrick Westphal ${ }^{2}$ e Elizabeth Santin ${ }^{2}$
}

\begin{abstract}
Muniz E.C., Pickler L., Lourenço M.C., Kraieski A.L., Mesa D., Westphal P. \& Santin E. 2015. [Evaluation of immune response of the intestinal mucosa in broilers challenged with different Salmonella serovars.] Avaliação da resposta imunológica da mucosa intestinal de frangos de corte desafiados com diferentes sorovares de Salmonella. Pesquisa Veterinária Brasileira 35(3):241-248. Laboratório de Microbiologia e Ornitopatologia, Departamento de Medicina Veterinária, Universidade Federal do Paraná, Rua dos Funcionários 1540, Curitiba, PR 80035-050, Brazil. E-mail: eduardo.muniz@ufpr.br

The study was designed to compare the effect of different Salmonella serovars in immune response across the count of CD8+ cells, CD4+ cells, goblet cells and macrophages in the gut mucosa of broilers. During the experimental inoculation at 7 day-old were used Salmonella enterica subspecies enterica sorovars Enteritidis, Typhimurium, Senftenberg, Mbandaka and Minnesota. It was observed that all serovars tested were capable of contaminating the poultry being possible counts of Salmonella in cloacal swabs, $48 \mathrm{~h}$ after inoculation and into the crop and cecum, at 14 and 20 day-old. Serovars tested had different effects on broiler performance assessed at 20 days. In the mucosa of the ileum and cecum of broilers, it was observed that some of the serotypes increased CD8 + cells, CD4 + cells, goblet cells and macrophages compared to the negative control group both at 14 and at 20 day-old. $S$. Enteritidis and $S$. Typhimurium are the serovars that showed the more intense effect in live performance and in the immune system of birds showing pathogenic characteristic; generally the broilers of the negative control showed significantly less immune cells on the intestinal mucosa than broilers inoculated experimentally. However, it was found that the Salmonella serovars used in this study had different effects on the cellular dynamics of the mucosa of the ileum and cecum and differently affect weight gain and average daily gain of poultry showing different levels of pathogenicity.
\end{abstract}

INDEX TERMS: Samonella, intestinal mucosa, broilers, immune response, immunohistochemistry

RESUMO.- Objetivou-se com o presente estudo comparar o efeito de diferentes sorovares de Salmonella na resposta imune local da mucosa do intestino de frangos de corte. Aos sete dias de idade, as aves foram desafiadas com os sorovares $S$. Enteritidis, $S$. Typhimurium, $S$. Senftenberg, $S$. Mbandaka e $S$. Minnesota. Foi observado que todos os sorovares testados foram capazes de colonizar o intestino das aves sendo pos-

\footnotetext{
${ }^{1}$ Recebido em 19 de junho de 2014.

Aceito para publicação em 23 de março de 2015.

${ }^{2}$ Laboratório de Microbiologia e Ornitopatologia, Departamento de Medicina Veterinária, Universidade Federal do Paraná (UFPR), Rua dos Funcionários 1540, Curitiba, PR 80035-050, Brasil. *Autor para correspondência: eduardo.muniz@ufpr.br
}

sível o isolamento de Salmonella em suabes de cloaca, $48 \mathrm{~h}$ após inoculação. De maneira geral, as aves do grupo controle negativo, que não foram desafiados apresentaram quantidade significativamente menor de células imunológicas na mucosa intestinal do que as aves desafiadas. Porém, verificou-se que os sorovares de Salmonella, utilizados neste estudo, apresentaram diferentes efeitos sobre a dinâmica celular da mucosa do íleo e ceco e afetaram de modo diferente o ganho de peso e ganho médio diário das aves demonstrando distintos graus de patogenicidade. Os sorovares Enteritidis e Typhimurium apresentaram um efeito mais intenso tanto no desempenho quanto na mobilização de células imunológicas na mucosa intestinal de frangos de corte. 
TERMOS DE INDEXAÇÃO: Salmonella, mucosa intestinal, frangos de corte, resposta imunológica, imuno-histoquímica.

\section{INTRODUÇÃO}

As salmoneloses estão entre as doenças potencialmente transmitidas pelos alimentos, sendo utilizadas como critério de qualidade dos produtos e interferindo no comércio internacional da carne de frango. Bactérias deste gênero têm sido identificadas como uma das principais causas de toxinfecções alimentares em seres humanos (Mürmann et al. 2008).

No relatório anual da Agência Nacional de Vigilância Sanitária Brasileira (ANVISA 2007), a Salmonella Enteritidis (SE) foi representada como o principal sorovar isolado em casos de toxinfecções em seres humanos com participação de 48,8\% do total reportado. A SE também foi o principal sorovar isolado em amostras de frangos de corte em abatedouro, realizadas pelo Ministério da Agricultura Pecuária e Abastecimento (MAPA) no Brasil (Freitas 2011). Neste levantamento também foi observado aumento no percentual de outros sorovares como, por exemplo, a Salmonella Minnesota encontrada em 9,38\% das amostras de abatedouro entre o período de 2009 e 2010.

Distintos sorovares também tem sido reportados na Europa, em carne de frango exportada do Brasil (RASFF 2012). Neste último ano os sorovares mais presentes tem sido Salmonella Heidelberg seguida de Salmonella Minnesota.

$\mathrm{O}$ controle das salmoneloses, como zoonose, representa um desafio à saúde pública em função da complexidade da sua epidemiologia, onde a prevalência dos diferentes sorovares pode variar entre localidades, estados e países, sendo recomendado estabelecer medidas de vigilância e identificação destes sorovares em seres humanos e aves de produção, com o propósito de desenvolver programas de controle específicos (Souza 2012).

Um fato relevante que dificulta o controle deste microrganismo na cadeia de produção de aves é a ausência de sinais clínicos e/ou lesões em lotes contaminados por sorovares paratifoides onde, na maioria das vezes, a ave é portadora assintomática (Freitas 2011). Além disso, estudo epidemiológico realizado por Rabsch et al. (2000) demonstraram que existe relação de exclusão competitiva entre diferentes sorovares. Este comportamento de alternância entre sorovares distintos na população indica que o nicho ecológico de um sorovar específico pode ser ocupado por outro. Dessa forma, tendo em vista a emergência de novos sorovares em determinadas áreas, o controle das salmoneloses passa pelo conhecimento dos comportamentos dos diferentes sorovares em relação a sua prevalência e característica de patogenicidade. Pesquisas comparativas têm demonstrado que os diferentes sorovares apresentam distintas patogenicidades quando comparados entre si (Gast \& Benson 1995).

A imuno-histoquímica é uma importante ferramenta de diagnóstico e pesquisa em avicultura, pois visa identificar as células responsáveis pela imunidade celular como linfócitos e macrófagos em aves desafiadas com Salmonella, por exemplo, e dessa forma melhor compreender a interação patógeno-hospedeiro (Jeurissen et al. 2002).
Com o objetivo de melhor entender como acontecem essas diferenças de resposta imunológica em frangos de corte, foi desenvolvido o presente estudo para comparar a infecção dos diferentes sorovares na resposta imune por meio da dinâmica de células imunológicas na mucosa intestinal de aves.

\section{MATERIAL E MÉTODOS}

\section{Animais e instalações}

Foram alojados 180 frangos de corte do primeiro ao 20 o dia de idade da linhagem Cobb 500, divididos em seis tratamentos em delineamento experimental inteiramente casualizado (Quadro 1).

As aves de cada tratamento foram alojadas em salas separadas, idênticas, localizadas lado a lado, com pressão negativa de forma a evitar a contaminação entre os diferentes tratamentos. Estas salas foram previamente limpas, desinfetadas e a cama de maravalha utilizada foi previamente esterilizada em autoclave à temperatura de $121^{\circ} \mathrm{C}$ durante 15 minutos. Foi realizado teste de esterilidade nas salas, equipamentos e cama por meio de suabes de arrasto antes do início do experimento, assim como foi realizado isolamento bacteriano no fígado e cecos de cinco aves para certificação da ausência de Salmonella.

Os animais foram mantidos em temperatura ideal de conforto para a idade das aves de acordo com orientação do manual de manejo Cobb, com fornecimento de água e ração ad libitum, isenta de antimicrobianos promotores de crescimento e coccidiostáticos sendo alimentadas com dietas fareladas e balanceadas em níveis iguais aos recomendados para idade das aves (Rostagno 2011). Para garantir a uniformidade, todas as aves foram pesadas individualmente e distribuídas igualmente de acordo com o peso inicial entre os tratamentos. Ao final do experimento ( $20^{\circ}$ dia de idade), as aves foram novamente pesadas para obtenção do cálculo do peso médio e ganho de peso diário nos diferentes tratamentos.

Este projeto foi aprovado pela Comissão de Ética no Uso de Animais (CEUA SCA) da Universidade Federal do Paraná sob Protocolo $\mathrm{n}^{\mathrm{0}} \mathrm{31}-2011$.

\section{Digestibilidade}

Foi realizado experimento de digestibilidade para determinar o coeficiente de retenção de matéria seca (MS), de extrato etéreo (EE), de proteína bruta (PB) e de energia bruta (EB) nas fezes ileais das aves. Foi realizada colheita de excretas do íleo no momento da morte das 20 aves por tratamento ( 5 pools de 4 aves) aos 20 dias. Para determinação da digestibilidade foi utilizada a metodologia de Sakomura \& Rostagno (2007). Em seguida, as amostras foram moídas e encaminhadas ao laboratório para análise de MS, EE, PB e EM segundo recomendações do método descrito por Silva \& Queiroz (2002). Os resultados foram submetidos à análise de variância e as diferenças entre as médias foram determinadas pelo teste de Tukey $(\mathrm{P}<0,05)$.

\section{Quadro 1. Descrição da fórmula antigênica dos diferentes sorovares de Salmonella utilizadas na inoculação experimental dos distintos grupos}

\begin{tabular}{|c|c|c|c|c|c|}
\hline \multirow{2}{*}{$\begin{array}{l}\text { Trata- } \\
\text { mento }\end{array}$} & \multirow[t]{2}{*}{ Sorovar/Grupo } & \multirow{2}{*}{$\begin{array}{c}\text { Antígeno } \\
\text { somático (0) }\end{array}$} & \multicolumn{3}{|c|}{ Antígeno flagelar $(\mathrm{H})$} \\
\hline & & & Fase 1 & Fase 2 & Outra \\
\hline $\mathrm{T} 1$ & Controle Negativo & - & - & - & \\
\hline $\mathrm{T} 2$ & $S$. Enteritidis $/ \mathrm{D}_{1}$ & $1,9,12$ & $\mathrm{~g}, \mathrm{~m}$ & - & \\
\hline T3 & $S$. Typhimurium/B & $1,4,[5], 12$ & $\mathrm{i}$ & 1,2 & \\
\hline $\mathrm{T} 4$ & S. Senftenberg/ $\mathrm{E}_{4}$ & $1,3,19$ & $\mathrm{~g},[\mathrm{~s}], \mathrm{t}$ & - & {$\left[\mathrm{z}_{27}\right],\left[\mathrm{z}_{34}\right],\left[\mathrm{z}_{37}\right],\left[\mathrm{z}_{43}\right]$,} \\
\hline T5 & S. Mbandaka $/ \mathrm{C}_{1}$ & $6,7,14$ & $\mathrm{z}_{10}$ & e,n,z15 & {$\left[\mathrm{z}_{37}\right],\left[\mathrm{z}_{45}\right]$} \\
\hline T6 & S. Minnesota/L & 21 & $\mathrm{~b}$ & $e, n, x$ & {$\left[\mathrm{z}_{33}\right],[\mathrm{z} 49]$} \\
\hline
\end{tabular}




\section{Cepa e inoculação}

Aos sete dias de idade as aves dos tratamentos foram inoculadas por via intra esofagiana com $1 \mathrm{~mL}$ de suspensão de Salmonella Enteritidis (SE), Salmonella Typhimurium (ST), Salmonella Senftenberg (SS), Salmonella Mbandaka (SMb) e Salmonella Minnesota (SM) na concentração $10^{5} \mathrm{UFC} / \mathrm{mL}$ de acordo com o Quadro 1. As cepas utilizadas no experimento foram isoladas de lotes de frangos de corte em condições de criação industrial.

Para o preparo do inóculo, as respectivas colônias de Salmonella foram retiradas do ágar estoque e incubadas em solução BHI (Brain Heart Infusion) por $24 \mathrm{~h}$ a $37^{\circ} \mathrm{C}$, separadamente. Em seguida, semeou-se a solução com um suabe em ágar Mueller Hinton por $24 \mathrm{~h}$ a $37^{\circ} \mathrm{C}$. A placa foi lavada, com solução fisiológica esterilizada. 0 líquido foi diluído até a concentração 0,5 da escala de MacFarland, que corresponde a concentração $10^{8}$ de UFC (Unidades Formadoras de Colônias). Fez-se diluições seriadas em tubos contendo $9 \mathrm{~mL}$ de solução fisiológica esterilizada até a concentração $10^{6} \mathrm{UFC} / \mathrm{mL}$ sendo posteriormente cultivada em ágar Mueller Hinton para determinar a contagem de unidades formadoras de colônias por mL (Pickler et al., 2012),

\section{Análises microbiológica, histopatológica e imuno-histoquímica}

Para análise microbiológica foram realizados suabes de cloaca 48h PI, sendo cinco amostras por tratamento (cada amostra foi composta por um pool de 3 animais) para análise de contagem de Salmonella. Aos 14 e 20 dias de idade (10 animais por tratamento/coleta) foram eutanaziados por deslocamento cervical posteriormente à insensibilização com barbitúricos e necropsiados para colheita de inglúvio e ceco de forma asséptica e posterior análise de Salmonella.

As análises de contagem de Salmonella foram realizadas de acordo com Pickler et al. (2012). Os suabes de cloaca, os inglúvios inteiros e os cecos com conteúdo foram macerados e diluídos em água peptonada a $2 \%$ em proporção de 1:10. Retirou-se $1 \mathrm{~mL}$ da solução de água peptonada $2 \%$ que foi acrescentado em um tubo contendo $9 \mathrm{~mL}$ de água peptonada $0,1 \%$ e assim sucessivamente até a diluição $10^{-3}$. Posteriormente retirou-se $100 \mu \mathrm{L}$ de cada diluição, espalhou-se o líquido em duplicata em placas de ágar Verde Brilhante com novobiocina e realizou-se a semeadura com alça de Drigalsky esterilizada. As placas foram incubadas em estufa regulada a $35 \pm 2^{\circ} \mathrm{C}$ por 24 h e submetidas à posterior contagem das colônias típicas.

A solução inicial de água peptonada $2 \%$ foi incubada a $35 \pm 2{ }^{\circ} \mathrm{C}$ por $24 \mathrm{~h}$. Em caso de não ter ocorrido crescimento de colônias típicas de Salmonella sp. em plaqueamento direto, retirou-se 100 $\mu \mathrm{L}$ da solução inicial em água peptonada $2 \%$ e acrescentou-se em um tubo contendo $10 \mathrm{~mL}$ de caldo Rappaport-Vassiliadis, incubou-se em estufa regulada a $42 \pm 2^{\circ} \mathrm{C}$ por $24 \mathrm{~h}$ para confirmação da positividade ou negatividade da amostra.

Os resultados das contagens de colônias foram expressos de acordo com Procedimentos de Contagem de Colônia de acordo com a Normativa no 62 publicada em 26 de agosto de 2003 (Brasil 2003). Os resultados foram transformados em Log10 para análise estatística. Amostras das colônias foram enviadas à Fiocruz para tipificação e confirmação dos sorovares testados.

Para análise de células caliciformes, foram realizadas coletas de fragmentos de íleo e ceco de cinco animais por tratamento e fixados em formol tamponado $10 \%$. Os tecidos foram processados rotineiramente para histologia e coradas com Alcian Blue. Foi avaliado o número de células caliciformes, com leitura de 20 campos por tratamento em aumento de 100x (Olympus BX41 Olympus America Inc., NY, USA) (Smirnov et al. 2004).

Para as análises de linfócitos T CD8+, CD4+ e macrófagos, amostras de íleo e ceco foram coletadas e incluídas em gel Tissue-Tek O.C.T. (Miles, Elkhart IN, US), congeladas em nitrogênio líquido, seccionadas com $5 \mu \mathrm{m}$ de espessura em aparelho criostato e fixadas em lâminas carregadas positivamente em acetona à $100 \%$. Em seguida foi realizada a re-hidratação com PBS 0,1M e pH 7,6, bloqueio da peroxidase endógena com peróxido de hidrogênio $3 \%$ por 5 minutos e proteína bloqueadora por 8 minutos. Os anticorpos primários utilizados foram Anti-CD8 (CT-8 Southern Biotech 1:100), Anti-CD4 (CT-4 Southern Biotech 1:100) e Anti-macrófago KUL01 Southern Biotech 1:150), incubados por 90 minutos a $37^{\circ} \mathrm{C}$. Para detecção da reação foram utilizados anticorpos secundários anti-camundongo e anti-coelho combinados em sistema de amplificação, kit ADVANCE $^{\circledR}$, por 30 minutos. Para revelação da reação foi utilizado cromógeno, kit $\mathrm{DAB}^{\circledR}$, por 30 segundos. As lâminas foram contra-coradas com Hematoxilina de Meyer, lavadas em água para posterior montagem das mesmas, adaptado de Jeurissen et al. (2002). Campos microscópicos com presença de células positivas foram quantificados em microscopia de luz com ampliação de 100x (Olympus BX41 Olympus America Inc., NY, USA). Foram analisados 20 campos para cada tratamento e para cada marcador de superfície celular.

\section{Análise estatística}

Para realização da análise estatística foi utilizado o programa estatístico StatView for Windows Copyright ${ }^{\circledR} 1998$ (SAS Institute Inc., NC, USA). As contagens de colônias de Salmonella foram transformadas em Log 10 para análise estatística. As variáveis foram analisadas inicialmente pelo teste de normalidade (Shapiro-Wilk). Todos os resultados foram submetidos à ANOVA, sendo aplicado o teste de Tukey a 5\% de probabilidade para as variáveis paramétricas (peso médio e digestibilidade) e o teste de Kruskal-Wallis à 5\% para as variáveis não paramétricas (microbiologia e imuno-histoquímica).

\section{RESULTADOS \\ Desempenho e digestibilidade}

O peso médio e ganho de peso diário das aves nos diferentes tratamentos ao primeiro e ao $20^{\circ}$ dia de idade estão expressos no Quadro 2. Foi possível observar que aves desafiadas com SM apresentaram maior peso vivo aos 20 dias e maior ganho de peso diário do primeiro ao $20 \cong$ dia quando comparadas a aves desafiadas com ST e SE.

Os resultados do percentual de digestibilidade de MS, EE, PB e EB das fezes ileais coletadas com 20 dias de idade das aves estão expressos no Quadro 3. Não houve diferença significativa entre os percentuais de digestibilidade de MS, EE e EB nos diferentes tratamentos. Já na digestibilidade da PB houve diferença significativa apenas entre o grupo inoculado com SMb, SS e o grupo controle negativo. Os demais

\section{Quadro 2. Peso médio e ganho de peso diário das aves (GPD) erro padrão nos diferentes grupos desafiados com Salmonella ao primeiro, e 20 dias de idade (Resultados Expressos em gramas)}

\begin{tabular}{lccc}
\hline Desafio das aves & Peso 1 dia & Peso 20 dias & GPD 1-20 dias \\
\hline Controle negativo & $43,80 \pm 2,20$ & $863,30 \pm 58,60^{\mathrm{ab}}$ & $40,92 \pm 2,89^{\mathrm{ab}}$ \\
Salmonella Enteritidis & $43,52 \pm 2,53$ & $858,16 \pm 129,20^{\mathrm{b}}$ & $40,74 \pm 6,47^{\mathrm{b}}$ \\
Salmonella Typhimurium & $43,72 \pm 2,46$ & $849,93 \pm 62,43^{\mathrm{b}}$ & $40,29 \pm 3,12^{\mathrm{b}}$ \\
Salmonella Senftenberg & $43,32 \pm 2,63$ & $888,27 \pm 71,24^{\mathrm{ab}}$ & $42,24 \pm 3,52^{\mathrm{ab}}$ \\
Salmonella Mbandaka & $43,55 \pm 2,51$ & $886,00 \pm 94,15^{\mathrm{ab}}$ & $42,13 \pm 4,66^{\mathrm{ab}}$ \\
Salmonella Minnesota & $43,55 \pm 2,25$ & $923,13 \pm 68,00^{\mathrm{a}}$ & $43,97 \pm 3,40^{\mathrm{a}}$ \\
Valor de P & 0,9700 & 0,0100 & 0,0000
\end{tabular}

$\overline{\mathrm{a}, \mathrm{b}}$ Letras diferentes na mesma coluna são significativamente diferentes pelo teste estatístico de Tukey $\mathrm{P} \leq 0,05$. 
tratamentos não apresentaram diferenças estatisticamente significativas.

\section{Microbiologia}

As amostras de fígado e ceco coletadas ao primeiro dia foram todas negativas para análise de Salmonella sp. 0 controle negativo não inoculado permaneceu negativo para Salmonella durante todo o período indicando a eficiência do sistema de isolamento entre as câmaras que separavam os tratamentos.

Quadro 3. Taxa de retenção de nutrientes de frangos de corte desafiados com Salmonella (Resultados expressos em $\%$ de digestibilidade)

\begin{tabular}{lcccc}
\hline Desafio das aves & MS & EE & PB & EB \\
\hline Controle negativo & 69,38 & 92,80 & $84,44^{\text {a }}$ & 70,49 \\
Salmonella Enteritidis & 69,30 & 92,74 & $82,47^{\text {ab }}$ & 70,70 \\
Salmonella Typhimurium & 68,61 & 91,73 & $82,51^{\text {ab }}$ & 69,74 \\
Salmonella Senftenberg & 70,54 & 91,97 & $84,26^{\text {a }}$ & 71,48 \\
Salmonella Mbandaka & 67,95 & 90,71 & $81,41^{\text {b }}$ & 69,49 \\
Salmonella Minnesota & 69,07 & 92,35 & $83,60^{\text {ab }}$ & 69,99 \\
Valor de P & 0,4077 & 0,0555 & 0,0119 & 0,5377 \\
CV & 2,71 & 1,19 & 1,65 & 2,53
\end{tabular}

$\overline{a, b}$ Letras diferentes na mesma coluna são significativamente diferentes pelo teste de Tukey $\mathrm{P} \leq 0,05$.

* MS = Matéria Seca, EE = Extrato Etéreo, PB = Proteína Bruta, EB = Energia Bruta.
Os resultados da contagem de colônias de Salmonella (média \pm erro padrão) em suabes de cloaca 48 h PI, inglúvios e cecos aos 14 e 20 dias de idade dos diferentes grupos estão expressos nos Quadros 4 e 5 . Todos os sorovares testados foram capazes de infectar significativamente as aves sendo possível a contagem dos respectivos sorovares em suabes 48 h PI e inglúvios e cecos aos 14 e 20 dias das aves.

\section{Histopatologia e imuno-histoquímica}

0 Quadro 6 expressa os resultados da contagem por campo com magnificação de 100x de linfócitos CD8+, CD4+, células caliciformes e macrófagos aos 14 e 20 dias de idade no íleo e ceco de frangos de corte nos diferentes trata-

Quadro 4. Média e erro padrão da contagem de Salmonella em suabes de cloaca realizados nos diferentes grupos $48 \mathrm{~h}$ após a inoculação (Resultados expressos em Log10)

\begin{tabular}{lc}
\hline Desafio das aves & Média $\pm \mathrm{EP}$ \\
\hline Controle negativo & $0,00 \pm 0,00^{\mathrm{a}}$ \\
Salmonella Enteritidis & $2,05 \pm 0,85^{\mathrm{b}}$ \\
Salmonella Typhimurium & $3,32 \pm 0,53^{\mathrm{c}}$ \\
Salmonella Senftenberg & $2,13 \pm 1,00^{\mathrm{b}}$ \\
Salmonella Mbandaka & $2,15 \pm 0,80^{\mathrm{b}}$ \\
Salmonella Minnesota & $2,22 \pm 0,61^{\mathrm{b}}$ \\
Valor de P & 0,001
\end{tabular}

$\overline{a, b, c}$ Letras diferentes na mesma coluna são significativamente diferentes pelo teste de Kruskal Wallis $\mathrm{P} \leq 0,05$.

Quadro 5. Média e erro padrão da contagem de Salmonella nos diferentes grupos em inglúvios e ceco aos 14 dias e 20 dias de idade das aves (Resultados expressos em Log 10)

\begin{tabular}{|c|c|c|c|c|c|c|c|}
\hline Fragmento/Idade & $\mathrm{n}$ & Controle & SE* & $\mathrm{ST}^{*}$ & SS* & $\mathrm{SMb}^{*}$ & $\mathrm{SM}^{*}$ \\
\hline Inglúvio/14 dias & 10 & $0,00 \pm 0,00^{\mathrm{a}}$ & $1,33 \pm 0,95^{b}$ & $1,62 \pm 0,72^{\mathrm{b}}$ & $2,23 \pm 1,44^{b}$ & $1,00 \pm 0,00^{\mathrm{b}}$ & $1,70 \pm 1,41^{b}$ \\
\hline Ceco/14 dias & 10 & $0,00 \pm 0,00^{\mathrm{a}}$ & $3,90 \pm 1,36^{c}$ & $3,64 \pm 1,35^{\mathrm{bc}}$ & $3,01 \pm 2,18^{\mathrm{bc}}$ & $2,00 \pm 1,75^{\mathrm{b}}$ & $4,35 \pm 1,53^{c}$ \\
\hline Inglúvio/20 dias & 10 & $0,00 \pm 0,00^{\mathrm{a}}$ & $0,87 \pm 0,35^{\mathrm{ab}}$ & $1,50 \pm 0,90^{\mathrm{b}}$ & $1,30 \pm 0,50^{\mathrm{b}}$ & $1,12 \pm 0,35^{\mathrm{b}}$ & $0,55 \pm 1,54^{\mathrm{ab}}$ \\
\hline Ceco/20 dias & 10 & $0,00 \pm 0,00^{\mathrm{a}}$ & $3,92 \pm 1,12^{c}$ & $2,15 \pm 1,34^{\mathrm{b}}$ & $3,36 \pm 2,00^{c}$ & $2,92 \pm 1,90^{\mathrm{bc}}$ & $2,97 \pm 1,83^{\text {bc }}$ \\
\hline Valor de P & 0,001 & & & & & & \\
\hline
\end{tabular}

$\overline{a, b, c}$ Letras diferentes na mesma linha são significativamente diferentes pelo teste de Kruskal Wallis $\mathrm{P} \leq 0,05$.

* SE = Salmonella Enteritidis, ST = Salmonella Typhimurium, SS = Salmonella Senftenberg, SMb = Salmonella Mbandaka, SM = Salmonella Minnesota.

Quadro 6. Média da contagem de células CD8+, CD4+, caliciformes e macrófagos no intestino das aves nos diferentes grupos aos 14 e 20 dias de idade (Resultados em unidades)

\begin{tabular}{|c|c|c|c|c|c|c|c|c|}
\hline Fragmento/Idade & Variável & $\mathrm{n}$ & Controle & $\mathrm{SE}^{*}$ & $\mathrm{ST}^{*}$ & $\mathrm{SS}^{*}$ & $\mathrm{SMb}^{*}$ & $\mathrm{SM}^{*}$ \\
\hline \multirow{3}{*}{ Íleo/14 dias } & CD8+ & 10 & $27,90^{\mathrm{b}}$ & $62,70^{\mathrm{a}}$ & $63,13^{\mathrm{a}}$ & $47,6^{\mathrm{ab}}$ & $34,63^{\mathrm{b}}$ & $37,03^{\mathrm{ab}}$ \\
\hline & $\mathrm{CD} 4+$ & 10 & $9,76^{c}$ & $69,80^{\mathrm{a}}$ & $67,46^{a}$ & $47,2^{\mathrm{ab}}$ & $32,03^{b c}$ & $46,66^{\mathrm{ab}}$ \\
\hline & Caliciformes & 20 & $34,82^{c}$ & $79,62^{\mathrm{ab}}$ & $99,10^{\mathrm{a}}$ & $32,7^{c}$ & $68,92^{\mathrm{ab}}$ & $47,85^{b c}$ \\
\hline \multirow{5}{*}{ Íleo/20 dias } & Macrófago & 10 & $12,26^{\mathrm{b}}$ & $54,86^{\mathrm{a}}$ & $62,7^{a}$ & $53,9^{a}$ & $53,63^{\mathrm{a}}$ & $35,53^{\mathrm{ab}}$ \\
\hline & $\mathrm{CD} 8+$ & 10 & 16,60 & 31,25 & 27,80 & 35,55 & 33,05 & 38,75 \\
\hline & CD4+ & 10 & $10,75^{c}$ & $39,10^{\mathrm{ab}}$ & $53,35^{a}$ & $30,1^{\mathrm{bc}}$ & $26,55^{b c}$ & $23,15^{b c}$ \\
\hline & Caliciforme & 20 & 68,55 & 78,52 & 64,90 & 49,7 & 53,87 & 47,42 \\
\hline & Macrófago & 10 & $6,70^{c}$ & $40,75^{\mathrm{bc}}$ & $46,60^{\mathrm{a}}$ & $29,5^{\mathrm{abc}}$ & $36,80^{\mathrm{ab}}$ & $22,60^{\mathrm{bc}}$ \\
\hline \multirow{3}{*}{ Ceco/14 dias } & $\mathrm{CD} 8+$ & 10 & $22,23^{b}$ & $62,46^{\mathrm{a}}$ & $75,50^{\mathrm{a}}$ & $32,3^{b}$ & $48,86^{\mathrm{b}}$ & $31,36^{b}$ \\
\hline & $\mathrm{CD} 4+$ & 10 & $28,50^{\mathrm{bc}}$ & $66,10^{\mathrm{a}}$ & $59,83^{a}$ & $44,1^{\mathrm{abc}}$ & $52,90^{\mathrm{a}}$ & $21,53^{c}$ \\
\hline & Caliciforme & 20 & $50,00^{\mathrm{bc}}$ & $93,05^{\mathrm{a}}$ & $72,32^{\mathrm{ab}}$ & $58,3^{\mathrm{bc}}$ & $52,22^{\mathrm{bc}}$ & $37,02^{c}$ \\
\hline \multirow{5}{*}{ Ceco/20 dias } & Macrófago & 10 & $27,83^{\mathrm{bc}}$ & $52,68^{\mathrm{ab}}$ & $71,53^{a}$ & $53,1^{\mathrm{ab}}$ & $51,86^{\mathrm{ab}}$ & $15,96^{c}$ \\
\hline & $\mathrm{CD} 8+$ & 10 & $29,30^{\mathrm{ab}}$ & $23,05^{b}$ & $47,00^{\mathrm{a}}$ & $47,2^{\mathrm{a}}$ & $19,55^{b}$ & $16,90^{\mathrm{b}}$ \\
\hline & CD4+ & 10 & $7,15^{c}$ & $45,20^{\mathrm{a}}$ & $47,45^{\mathrm{a}}$ & $30,8^{\mathrm{ab}}$ & $20,20^{\mathrm{bc}}$ & $32,20^{\mathrm{ab}}$ \\
\hline & Caliciforme & 20 & $51,27^{\mathrm{bc}}$ & $91,87 a$ & $72,20^{\mathrm{ab}}$ & $49,9^{\mathrm{bc}}$ & $62,10^{\mathrm{abc}}$ & $35,62^{c}$ \\
\hline & Macrófago & 10 & $21,70^{\mathrm{bc}}$ & $45,10^{\mathrm{a}}$ & $22,80^{\mathrm{abc}}$ & $45,6^{a}$ & $35,75^{\mathrm{ab}}$ & $12,00^{c}$ \\
\hline
\end{tabular}

$\overline{a, b, c}$ Letras diferentes na mesma linha são significativamente diferentes pelo teste de Kruskal Wallis P $\leq 0,05$.

* SE = Salmonella Enteritidis, ST = Salmonella Typhimurium, SS = Salmonella Senftenberg, SMb = Salmonella Mbandaka e SM = Salmonella Minnesota. 
mentos. As Figuras 1 e 2 demonstram cortes histológicos do ceco das aves com aplicação da técnica de imuno-histoquímica para células caliciformes e células CD8+, respectivamente.

$\mathrm{Na}$ análise de células CD8+ no íleo aos 14 dias de idade, aves desafiadas com SE e ST apresentaram quantidade significativamente maior de células quando comparado as aves desafiadas com SMb ou não desafiadas. Aos 20 dias de idade, não foram observadas diferenças estatisticamente significativas entre os tratamentos, no íleo. No ceco aos 14 dias de idade, foi observada quantidade significativamente maior de células CD8+ nos grupos desafiados com SE e ST quando comparado aos demais tratamentos. E aos 20 dias de idade no ceco, os grupos desafiados com ST e SS apresentaram contagem significativamente maior de células CD8+ com relação aos demais grupos exceto o controle.

No íleo, aos 14 e aos 20 dias de idade, as aves desafiadas com SE e ST apresentaram contagem significativamente maior de células CD4+ que o grupo controle. A imagem microscópica das células CD4+ eram semelhantes ao encontrado na Figura 2. No ceco, aos 14 dias os grupos desafiados

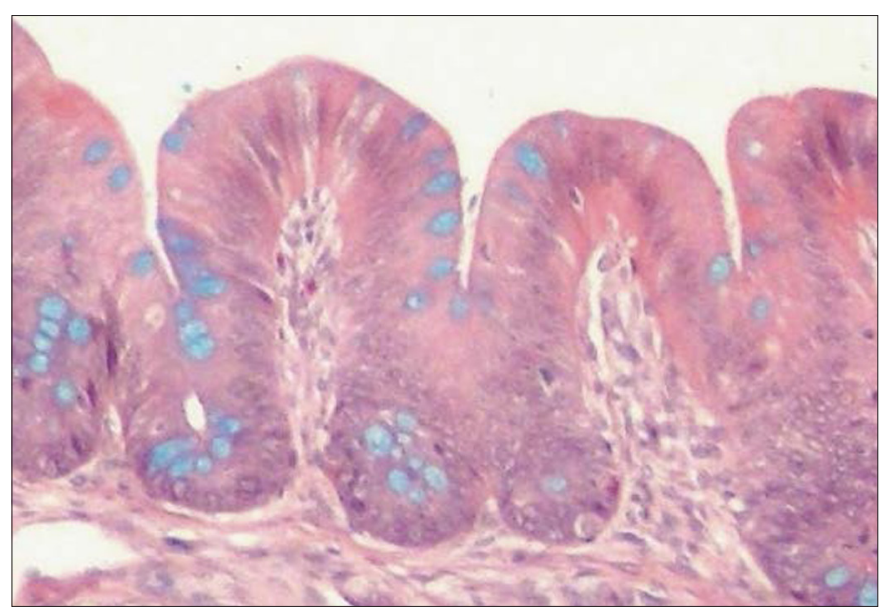

Fig. 1 Fotomicrografia dos cecos das aves com 20 dias de idade demonstrando as células caliciformes na mucosa intestinal e coradas em azul. Alcian Blue, 40x.

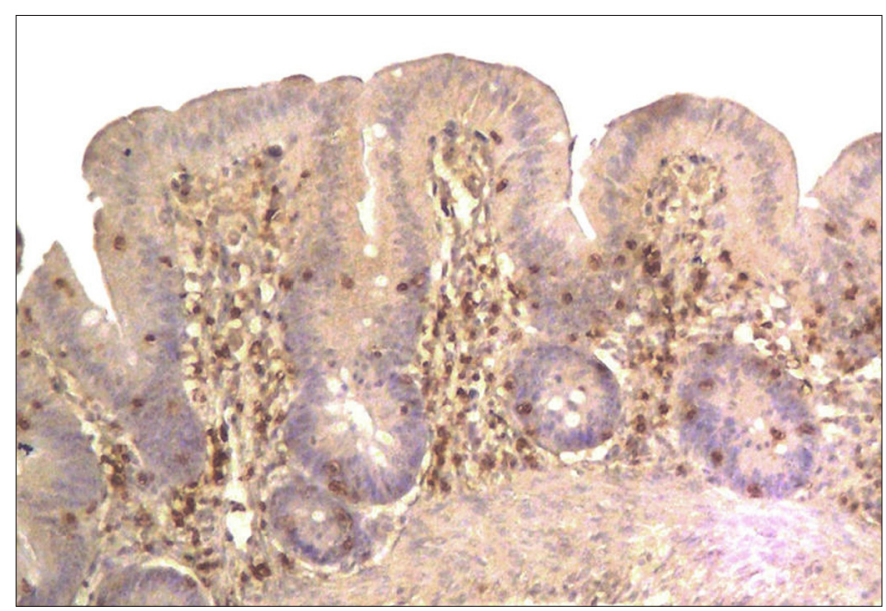

Fig. 2 Fotomicrografia dos cecos de aves com 20 dias de idade demonstrando as células CD8+ na mucosa intestinal e coradas em marrom. Imuno-histoquimica, 40x. com SE, ST e SMb apresentaram quantidade significativamente maior de células CD4+ quando comparado ao controle negativo e ao grupo desafiado com SM. Aos 20 dias, no ceco, as aves desafiadas com SE, ST, SS e SM apresentaram quantidade significativamente maior de células CD4+ que o grupo controle.

Com relação a contagem de células caliciformes, aos 14 dias de idade no íleo, as aves dos grupos não desafiados e desafiados com SS apresentaram contagem significativamente menor que os grupos com SE, ST e SMb. Aos 20 dias no íleo não foram observadas diferenças significativas entre os grupos. No ceco aos 14 e aos 20 dias de idade os grupos desafiados com SE e ST apresentaram contagem de células caliciformes significativamente maiores quando comparada ao grupo desafiado com SM.

Na análise de macrófagos na mucosa intestinal, aos 14 dias de idade no íleo, o grupo controle negativo apresentou quantidade significativamente menor de células que os demais grupos desafiados exceto o grupo SM. Aos 20 dias no íleo, o controle negativo apresentou quantidade significativamente menor de macrófagos que os grupos desafiados com ST e SMb. E aos 14 e 20 dias de idade, aves do grupo desafiado com SM, apresentaram quantidade significativamente menor de macrófagos nos cecos do que os grupos desafiados com SE, SS e SMb.

\section{DISCUSSÃO}

De maneira geral, a infecção por salmonelas paratíficas não interferem de forma significativa no desempenho zootécnico das aves, pois este grupo de salmonelas está altamente adaptado às aves e convivem de forma equilibrada com o hospedeiro. Porém, alguns sorovares, como por exemplo, a SE, tem extensa colonização no trato digestório e causam doença com envolvimento do sistema imunológico (Gast \& Benson 1995).

A avaliação de retenção e metabolizabilidade tem sido utilizada em experimentos de nutrição para avaliar a digestibilidade do alimento (Ravindran et al. 1999). No presente estudo investigou-se as diferenças na digestibilidade e desempenho das aves desafiadas com os diferentes sorovares. Com relação ao peso das aves aos 20 dias de idade nos diferentes tratamentos, o que mais se destaca foi o menor peso de aves inoculadas com SE e ST quando comparadas as aves desafiadas com SM, o que pode ser relacionado com o custo da resposta imunológica destas aves desafiadas com sorotipos de Salmonella muito imunogênicos como SE e ST (Berndt \& Methner 2001).

Nestes casos a resposta do organismo é a produção aumentada de células e proteínas, como as proteínas de fase aguda sintetizadas pelo fígado (Klasing 2007) e consequente piora no desempenho zootécnico. Este aumento na produção de células como resposta imunológica resulta em redistribuição e maior gasto de nutrientes como lisina. Estima-se que nas aves submetidas a desafio, a resposta imunológica inata e adaptativa utilizem $550 \mu \mathrm{mol} / \mathrm{kg} / \mathrm{dia}$ de lisina, a qual poderia ser utilizada para o crescimento animal sendo convertida em 7,8g de massa corporal/kg de peso vivo (Klasing 2004).

De acordo com Ravindran et al. (1999), houve consen- 
so que o método de colheita de conteúdo ileal ao invés da colheita total de excretas é o método mais eficiente para avaliação da digestibilidade dos alimentos em aves. Isso se deve ao fato de que a colheita neste ponto do intestino neutraliza o efeito adverso da microbiota cecal na digestibilidade da proteína. Utilizando esta metodologia, no presente estudo, verificou-se maior digestibilidade da proteína no grupo controle comparado ao grupo desafiado com SMb. Como este achado não está relacionado a piora no ganho de peso deste grupo desafiado com SMb, poderia ser relacionado a maior perda de integridade intestinal neste grupo, entretanto seria necessário mais estudos para entender estes resultados.

A Salmonella é considerada um patógeno intracelular facultativo e a imunidade mediada por células tem um papel muito importante para a eliminação da bactéria dos tecidos, com a participação de células T (Holt et al. 2010). Outros estudos avaliaram a interação dessas células em agregados linfoides intestinais após infecção oral com Salmonella em aves jovens (Sasai et al. 2000, Berndt et al. 2007, Hemert et al. 2007). As células T CD8+ desempenham um importante papel no combate das infecções primárias por salmonelas, assim como a resposta por IgA e leucócitos polimorfonucleados (Berndt \& Methener 2001).

No presente estudo foi observado que aves desafiadas com SE e ST apresentaram maior contagem de células CD4+ no íleo e ceco das aves aos 14 e 20 dias, quando comparado aos demais sorovares e o controle negativo, o que também foi observado em outros estudos onde foi evidenciado maior expressão destas células após infecção oral com SE (Sasai et al. 2000, Asheg et al. 2002) e com ST (Berndt \& Methner 2001, Beal et al. 2004). Em contrapartida, Hemert et al. (2007) mostraram diminuição na contagem destas células no jejuno ao primeiro e ao quinto dia pós-inoculação oral de 0,2 ml de $10^{5} \mathrm{UFC} / \mathrm{ml}$ de Salmonella Enteritidis quando comparado com o controle negativo.

O número de células CD8+ também foi maior no íleo e ceco de aves desafiadas com SE e ST quando comparado com os demais tratamentos. Berndt \& Methner (2001) observaram em frangos inoculados com ST, aumento destas células no ceco nos primeiros 9 dias PI comparado com o controle. Observa-se que aves desafiadas com SE apresentam aumento na quantidade de linfócitos T CD3+ (Pickler et al. 2013) e CD8+ (Berndt et al. 2006) na mucosa intestinal e redução dessas células na circulação sanguínea concluindo que rapidamente essas células migram do sangue ao intestino, primeiro sítio de infecção da Salmonella.

A maior expressão celular na mucosa intestinal dos frangos desafiados é devido à alta patogenicidade e capacidade imunogênica que possuem SE e ST (Hassan et al. 1991, Desmidt et al. 1997). Durante a infecção intestinal estes dois sorovares penetram nos enterócitos resultando em uma resposta inflamatória considerável, ao contrário da Salmonella Pullorum e Gallinarum que geram uma resposta inflamatória muito menor no intestino e maior em outros tecidos que colonizam (Barrow et al. 2012). Os sorovares hospedeiro-específicos persistem mais nos linfonodos mesentéricos, no caso de mamíferos, e são determinantes para sua virulência sistêmica (Paulin et al. 2002).
Em suínos inoculados oralmente com cepas de Salmonella Typhimurium e de Salmonella Choleraesuis, observou-se elevada contagem do sorovar Choleraesuis em linfonodos mesentéricos, enquanto o sorovar Typhimurium é associado com a rápida multiplicação na mucosa intestinal. Outra observação foi de que animais inoculados com ST apresentaram maior excreção que os desafiados com Choleraesuis. As diferenças também podem ser constatadas na resposta de citocinas, às $24 \mathrm{~h}$ PI, a ST provocou altos níveis de transcrição de TNF, IL-8 e IL-18 comparado com o sorovar Choleraesuis, de tal maneira que estes elevados níveis de citocinas pro-inflamatórias podem afetar à microbiota do intestino e promover a persistência intestinal da bactéria patogênica (Paulin et al. 2007).

A rápida multiplicação da ST poderia desencadear a primeira resposta inflamatória seguida de uma infecção de grande magnitude, isso confinaria localmente o processo à mucosa intestinal, enquanto que a lenta multiplicação do sorovar Choleraesuis lhe permitiria evadir da ativação da resposta imune inata, favorecendo sua disseminação (Uthe et al. 2007).

Os macrófagos tem uma importante função na defesa contra Salmonella. Em testes in vitro, essas células são capazes de fagocitar a Salmonella e prevenir sua multiplicação por meio de espécies reativas de oxigênio e nitrogênio (Shiloh \& Nathan 2000). Em trabalho realizado com cultivo celular Qureshi et al. (2000) observaram que macrófagos de aves foram capazes de eliminar efetivamente ST. No presente estudo, realizado in vivo, observou-se aumento significativo destas células nos tratamentos inoculados com SE e ST, sendo que nossos dados concordam com o trabalho de Berndt \& Methner (2004), que observaram grande quantidade de macrófagos no ceco de aves infectadas com ST entre os dias 4 e 12, com um pico de expressão celular ao quinto dia PI e com Van Immerseel et al. (2002) que observaram invasão de macrófagos na lâmina própria do ceco após inoculação com SE.

No presente estudo, aves inoculadas com SM apresentaram o maior peso aos 20 dias de idade quando comparada aos demais grupos. Estes resultados chamam a atenção, pois, além de inéditos, são contrários ao consenso geral de que toda Salmonella traz algum prejuízo ao desempenho dos animais contaminados independentemente do sorovar. Além disso, os resultados de células CD4+, CD8+ e macrófagos no intestino parecem mostrar que este sorotipo é pouco imunogênico, podendo se adaptar muito bem à microbiota intestinal, promovendo pouca resposta imunológica local, fato este que merece mais estudos para melhor compreensão da resposta imunológica de aves à SM e entendimento da eventual condição de equilíbrio que este microrganismo estabelece com o hospedeiro.

A composição antigênica utilizada para caracterizar os sorovares (Quadro 1) demonstra que a SM difere significativamente dos demais sorovares, pois não compartilha nenhum antígeno tanto somático quanto flagelar com os demais sorovares deste estudo. Isso é um indicativo da razão pela qual este sorovar pouco induz resposta na mucosa do intestino das aves.

Já os sorovares SS e SMb apresentaram comportamento 
intermediário em relação a SE, ST e SM, tanto na capacidade de estimular o sistema imunológico quanto na interferência em peso aos 20 dias. Eles assumiram posição média na imunogenicidade e no efeito em desempenho das aves inoculadas. Estes dados estão de acordo com o trabalho comparativo de Gantois et al. (2008) que indicaram maior capacidade de colonização dos órgãos reprodutores da SE e ST em relação a outros sorovares estudados. Aparentemente alguns sorovares possuem características particulares que aumentam a chance de infectar as aves causando perdas zootécnicas levando a um maior estímulo do sistema imune da ave.

Conclui-se que existe uma relação entre a intensidade de estímulo do sistema imunológico e o gasto energético dos animais, sendo que microrganismos mais patogênicos repercutem em menor ganho de peso das aves em função do custo para a formação da defesa linfocitária de mucosa. Além disso, os sorovares de Salmonella estudados demostraram diferentes efeitos sobre a dinâmica celular da mucosa do íleo e ceco e afetam de modo diferente o ganho de peso e ganho médio diário das aves. Mais estudos avaliando as respostas imunológicas precoces com avaliação de citocinas precisam ser conduzidos para se compreender melhor esses resultados.

\section{REFERÊNCIAS}

ANVISA 2007. Relatório Anual. Agência Nacional de Vigilância Sanitária. Disponível em <http://www.anvisa.gov.br/alimentos/Relatorio_GGALI_2007.pdf> Acessado em 16 ago. 2013.

Asheg A., Levkut M., Revajová V., Ševčíková Z., Kolodzieyski L. \& Pistl J. 2002. T lymphocyte subpopulations and B lymphocyte cellsin caecum and spleen of chicks infected with Salmonella Enteritidis. Acta Histochemica 104:435-439.

Barrow P.A., Jones M.A., Smith A.L. \& Wigley P. 2012. The long view: Salmonella, the last forty years. Avian Pathol. 41:413-420.

Beal R.K., Powers C., Wigley P., Barrow P.A. \& Smith A.L. 2004. Temporal dynamics of the cellular, humoral and cytokine responses in chickens during primary and secondary infection with Salmonella enterica serovar Typhimurium. Avian Pathol. 33:25-33.

Berndt A. \& Methner U. 2001. Gamma/delta T cell response of chickens after oral administration of attenuated and non-attenuated Salmonella Typhimurium strains. Vet. Immunol. Immunopathol. 78:143-161.

Berndt A. \& Methner U. 2004. B cell and macrophage response in chicks after oral administration of Salmonella Typhimurium strains. Comp. Immunol. Microbiol. Infect. Dis. 27:235-246.

Berndt A., Pieper J. \& Methner U. 2006. Circulating $\gamma \delta$ T cells in response to Salmonella enterica serovar Enteritidis exposure in chickens. Infect. Immun. 74:3967-3978.

Berndt A., Wilhelm A., Jugert C., Pieper J., Sachse K. \& Methner U. 2007. Chicken cecum immune response to Salmonella enterica serovars of different levels of invasiveness. Infect. Immun. 75:5993-6007.

Brasil 2003. Instrução Normativa nำ2 publicada em 26 de agosto de 2003. Oficializa os Métodos Analíticos Oficiais para Análises Microbiológicas para Controle de Produtos de Origem Animal e Água. Ministério da Agricultura Pecuária e Abastecimento, Brasília, DF.

Desmidt M., Ducatelle R. \& Haesebrouck F. 1997. Pathogenesis of Salmonella Enteritidis phage type four after experimental infection of young chickens. Vet. Microbiol. 56:99-109.

Freitas J. 2011. Evolução de sorovares Modelo de Banco de Cepas. Seminário Internacional de Salmonellose Aviar, Rio de Janeiro.

Gantois I., Eeckhaut V., Pasmans F., Haesebrouck F., Ducatelle R. \& Van Immerseel F. 2008. A comparative study on the pathogenesis of egg contamination by different serotypes of Salmonella. Avian Pathol. 37:399-406.
Gast R.K. \& Benson S.T. 1995. The comparative virulence for chicks of Salmonella enteritidis phage type 4 isolates and isolates of phage types commonly found in poultry in the United States. Avian Dis. 39:567-574.

Hassan J.O., Mockett A.P.A., Catty D. \& Barrow P.A. 1991. Infection and reinfection of chickens with Salmonella Typhimurium: Bacteriology and immune responses. Avian Dis. 35:809-819.

Hemert S.V., Hoekman A.J.W., Smits M.A. \& Rebel J.M.J. 2007. Immunological and gene expression responses to a Salmonella infection in the chicken intestine. Vet. Res. 38:51-63.

Holt P.S., Vaughn L.E \& Gast R.K. 2010. Flow cytometric characterization of Peyer's patch and cecal tonsil T lymphocytes in laying hens following challenge with Salmonella enterica serovar Enteritidis. Vet. Immunol. Immunopathol. 133:276-281.

Jeurissen S., Lewis F., Van der Klis J.D., Mroz Z., Rebel J. \& Huurne A. 2002. Parameters and techniques to determine intestinal health of poultry as constituted by immunity, integrity, and functionality. Curr. Issues Intest. Microbiol. 3:14-30.

Klasing K.C. 2004. The costs of immunity. Acta Zool. Sinica 50:961-969.

Klasing K.C. 2007. Nutrition and the immune system. Brit. Poult. Sci. 48: 525-537.

Mürmann L., Santos M.C.D., Longaray S.M., Both J.M.C. \& Cardoso M. 2008. Quantification and molecular characterization of Salmonella isolated from food samples involved in salmonellosis outbreaks in Rio Grande do Sul, Brazil. Braz. J. Microbiol. 39:529-534.

Paulin S.M., Jagannathan A., Campbell J., Wallis T.S. \& Stevens M.P. 2007. Net replication of Salmonella enterica serovars Typhimurium and Choleraesuis in porcine intestinal mucosa and nodes is associated with their differential virulence. Infect. Immunol. 75:3950-3960.

Paulin S.M., Watson P.R., Benmore A.R., Stevens M.P., Jones P.W., Villarreal-Ramos B. \& Wallis T.S. 2002. Analysis of Salmonella enterica serotype-host specificity in calves: Avirulence of S. enterica serotype Gallinarum correlates with bacterial dissemination from mesenteric lymph nodes and persistence in vivo. Infect. Immunol. 70:6788-6797.

Pickler L., Beirão B.C.B., Hayashi R.M., Durau J.F., Lourenço M.C., Caron L.F. \& Santin E. 2013. Effect of sanguinarine in drinking water on Salmonella control and the expression of immune cells in peripheral blood and intestinal mucosa of broilers.J. Appl. Poult. Res. 22:430-438.

Pickler L., Hayashi R.M., Lourenço M.C., Miglino L.B., Caron L.F., Beirão B.C.B., Silva A.V.F. \& Santin E. 2012. Avaliação microbiológica, histológica e imunológica de frangos de corte desafiados com Salmonella Enteritidis e Minnesota e tratados com ácidos orgânicos. Pesq. Vet. Bras. 32:27-36.

Qureshi M.A., Heggen C.L. \& Hussain I. 2000. Avian macrophage: effector functions in health and disease. Develop. \& Comp. Immunol. 24:103-119.

Rabsch W., Hargis B.M., Tsolis R.M., Kingslay R.A., Hinz K., Tschäpe H. \& Bäumler A. 2000. Competitive Exclusion of Salmonella Enteritidis by Salmonella Gallinarum in poultry. Emerg. Infec. Dis. 6:443-448.

Rasff. 2012. The rapid alert system for food and feed. Annual Report of European Comission. 60p. Disponível em <http://ec.europa.eu/food/ food/rapidalert/docs/rasff_annual_report_2012_en.pdf> Acesso em 18 dez. 2013.

Ravindran V., Hew L.I., Ravindran G. \& Bryden W.L. 1999.A comparison of ileal digesta and excreta analysis for the determination of amino acid digestibility in feed ingredients for poultry. Brit. Poult. Sci. 40:266-274.

Rostagno H.S. 2011. Tabelas Brasileiras para aves e suínos. Universidade Federal de Viçosa, Viçosa, MG.

Sasai K., Aita M., Lillehoj H.S., Miyamoto T., Fukata T. \& Baba E. 2000. Dynamics of lymphocyte subpopulation changes in the cecal tonsils of chickens infected with Salmonella Enteritidis. Vet. Microbiol. 74:345351.

Sakomura N.K. \& Rostagno H.S. 2007. Métodos de pesquisa e nutrição de monogástricos. Funep, Jaboticabal, SP, p.92-129.

Shiloh M.U. \& Nathan C.F. 2000. Reactive nitrogen intermediates and the pathogenesis of Salmonella and mycobacteria. Curr. Opin. Microbiol. 3:35-42.

Silva D.J. \& Queiroz A.C. 2002. Análise de Alimentos: Métodos químicos e biológicos. $3^{\text {a }}$ ed. UFV, Viçosa. 235p. 
Smirnov A., Sklan D. \& Uni Z. 2004. Mucin dynamics in the chick small intestine are altered by starvation. J. Nutr. 134:736-742.

Souza A. 2012. Introduction to the special issue: Salmonella in foods: evolution, strategies and challenges. Food Res. Int. 45:451-454.

Uthe J.J., Royaee A., Lunney J.K., Stabel T.J., Zhao S.H., Tuggle C.K. \& Bearson S.M.D. 2007. Porcine differential gene expression in response to Salmo- nella enterica serovars Choleraesuis and Typhimurium. Mol. Immunol. 44:2900-2914.

Van Immerseel F., De Buck J., De Smet I., Mast J., Haesebrouck F. \& Ducatelle R. 2002. Dynamics of immune cell infiltration in the caecal lamina propria of chickens after neonatal infection with a Salmonella Enteritidis strain. Develop. Comp. Immunol. 26:355-364. 